\title{
A influência de fatores emocionais sobre a hipertensão arterial
}

\author{
The influence of emotional factors on the arterial hypertension
}

Fabiana de Cássia Almeida Fonseca', Renata Zumerle Coelho1, Rodrigo Nicolato², Leandro Fernandes Malloy-Diniz ${ }^{3}$, Humberto Corrêa da Silva Filho ${ }^{4}$

\section{RESUMO}

Objetivo: Realizar revisão bibliográfica de artigos que abordam a relação entre hipertensão arterial e fatores emocionais, levando em consideração a relevância do tema. Métodos: Fezse busca ativa na Biblioteca Virtual em Saúde, na base de dados MedLine (1997-2008), utilizando palavras da língua portuguesa. Os descritores de assunto escolhidos foram "hipertensão" e "doença cardíaca coronária". Em seguida, refinou-se a busca com os termos "hostilidade", "raiva", "ansiedade", "comportamento impulsivo" e "personalidade impulsiva". Não foram selecionados artigos que tratavam exclusivamente de doenças cardiovasculares e fatores psicológicos ou que associavam hipertensão e doenças cardiovasculares com depressão e doença de Alzheimer. Resultados e discussão: Há inconsistência nos achados que relacionam os fatores emocionais com a hipertensão arterial e cardiopatias. Foram encontrados tanto estudos que demonstram relação positiva da raiva, hostilidade, ansiedade, impulsividade e estresse com hipertensão e doenças cardiovasculares quanto estudos que retratam relações

\section{Palavras-chave}

Hipertensão, doença cardíaca coronária, estresse, hostilidade/raiva. negativas. Conclusão: $\bigcirc$ que se pode inferir das relações pesquisadas é que o risco de desenvolvimento da hipertensão arterial e a reatividade cardiovascular parecem ser influenciados por fatores emocionais como impulsividade, hostilidade, estressores, ansiedade e raiva. No entanto, mais estudos são necessários para melhor elucidar essas relações.

\begin{abstract}
Objective: Conduct a bibliographic review of articles that deal a relationship between hypertension and emotional factors considering relevance of the subject. Methods: Through an active search in the Virtual Health Library, querying the database MedLine (1997-2008), we used words of the Portuguese language. The chosen descriptors are "hypertension" and "coronary heart disease". After that, the search was improved by the adding of terms "hostility", "anger", "anxiety", "impulsive behavior" and "impulsive personality". All articles that had an exclusive reference about cardiovascular diseases and psychological factors or article that associated hypertension and cardiovascular disease with depression and Alzheimer's disease were not selected. Results: There are inconsistencies in the findings about emotional factors related to hypertension and
\end{abstract}

1 Universidade Fundação Mineira de Educação e Cultura (Fumec).

2 Universidade Federal de Ouro Preto (UFOP), Departamento de Ciências Médicas, Instituto de Previdência dos Servidores do Estado de Minas Gerais (IPSEMG).

3 Universidade Federal de Minas Gerais (UFMG), Faculdade de Filosofia e Ciências Humanas, Departamento de Psicologia.

4 Universidade Federal de Minas Gerais (UFMG), Faculdade de Medicina, Departamento de Saúde Mental.

Endereço para correspondência: Renata Zumerle Coelho

Rua João Ferreira de Aguiar, 43/601, Fonte Grande - 32013-550 - Contagem, MG

Telefone: (031) 9141-0001

E-mail:zumerle@hotmail.com 
heart diseases. We found studies that demonstrate both positive and negative relationship between anger, hostility, anxiety, impulsivity and stress with hypertension and cardiovascular di-

\section{Keywords}

Hypertension, coronary

heart disease, stress,

hostility/anger. sease. Conclusion: We can infer from the searched relations is that the risk of hypertension's development and the cardiovascular reactivity seems to be influenced by emotional factors, like impulsivity, hostility, stressors, anxiety, anger. However, it is necessary to execute more studies to better elucidate these relations.

\section{INTRODUÇÃO}

A hipertensão arterial (HA) faz parte do grupo de doenças cardiovasculares que representam o maior percentual de causas de mortalidade por doenças como acidente vascular cerebral (AVC) e infarto agudo do miocárdio. Em 2001, as doenças do aparelho circulatório representaram 27\% dos óbitos no Brasil, com a região Sudeste apresentando 29,6\%. No Estado de Minas Gerais, as doenças cardiovasculares representaram 30,1\% dos óbitos por causa básica'. A hipertensão arterial constitui um agravo à saúde e sua prevalência na população brasileira adulta varia entre 15\% e 20\% e aumenta progressivamente com a idade ${ }^{2}$.

Essa entidade clínica multifatorial é caracterizada pela presença de níveis de pressão arterial sistólica (PAS) persistentemente iguais ou acima de 140 mmHg e/ou níveis de pressão arterial diastólica (PAD) persistentemente iguais ou acima de 90 mmHg. Operacionalmente, existem dois critérios reconhecidos para classificar indivíduos normotensos e hipertensos: o da Organização Mundial da Saúde (OMS)³, que estabelece pontos de corte em $95 \mathrm{mmHg}$ para a pressão arterial diastólica e $160 \mathrm{mmHg}$ para a pressão arterial sistólica, e o do Joint National Committee (JNC) ${ }^{4}$, com pontos de corte em 90 $\mathrm{mmHg}$ para a PAD e $140 \mathrm{mmHg}$ para a PAS. Em relação à definição de normotensão limítrofe e de hipertensão, o JNC apresentou um critério para definir pressão arterial normal-alta, de 130-139 mmHg para a PAS e 85 a $89 \mathrm{mmHg}$ para a PAD.

A hipertensão arterial pode ser primária/essencial ou secundária. As causas da hipertensão arterial primária não são conhecidas na maioria dos casos, já a hipertensão arterial secundária deve ser investigada, uma vez que o diagnóstico etiológico significa, em muitos casos, a possibilidade de tratamento específico e cura ou controle por intervenção clínica ou cirúrgica 5 .

Quanto aos fatores de risco conhecidos para a HA, os mais importantes são: obesidade, fumo, ingestão de álcool, história familiar de hipertensão, fatores psicológicos, certos traços de personalidade e estresse, que podem ser importantes desencadeadores no desenvolvimento da hipertensão ${ }^{6-9}$. Muriel et al.10 acrescentam, ainda, como fatores de risco, a genética e os fatores ambientais (obesidade, inatividade física e abundância no consumo de sódio).
Por causa da relevância do tema, muito se tem estudado sobre o assunto, embora pouca atenção tenha sido dada à associação dos fatores emocionais (raiva, hostilidade, impulsividade, ansiedade) com a pressão arterial. Os estudos iniciais sobre o assunto se deram pela abordagem da personalidade tipo A. Em 1957, Rosenman et al."1, cardiologistas do hospital Monte Sinai em São Francisco, Califórnia, estabeleceram um estilo de comportamento que chamaram de padrão de conduta tipo A, que constitui um fator de risco para a cardiopatia isquêmica. Esse padrão de conduta, segundo Rosenman et al."1, caracteriza-se por alta competitividade, impulsividade e agressividade. Eles sugeriram que esse seria um fator de risco independente e com potencial de predição aproximada aos fatores de risco clássicos das doenças coronarianas como fumo, pressão arterial elevada e taxas elevadas de colesterol. Atualmente, é sabido que o tipo A de personalidade não correspondeu às expectativas esperadas, mas foi um importante passo no estudo dessa correlação.

Na tentativa de averiguar melhor o ceticismo de estudos posteriores sobre o envolvimento do tipo A de personalidade e doenças cardiovasculares, Gallacher et al.12, em um universo de 2.394 pessoas pesquisadas em um período de cinco anos, concluiu a potencialidade do tipo A como gatiIho para eventos isquêmicos coronarianos, mas com ressalvas e necessidade de mais estudos futuros.

Atualmente, Muriel et al. ${ }^{10}$ propuseram um estudo que analisa a relação da ansiedade com a personalidade tipo A - descrita como de maior vulnerabilidade ao estresse e à gênese da hipertensão arterial. Outros estudos apontam como a associação entre expressão da raiva e níveis de pressão arterial pode ser afetada por vários fatores.

De acordo com descrições neuroanatômicas, verifica-se que as áreas encefálicas relacionadas com o comportamento emocional ocupam territórios muito amplos do telencéfalo e do diencéfalo, nos quais se encontram as estruturas que integram o sistema límbico, a área pré-frontal e o hipotálamo. Cabe ressaltar o papel dessas áreas na regulação das atividades viscerais por intermédio do sistema nervoso autônomo $(\mathrm{SNA})^{13}$.

Levando-se em consideração o aspecto neurobiológico, pode-se apontar relação entre o funcionamento do sistema nervoso simpático (SNS), as emoções e a hipertensão arterial. O SNA, também conhecido como involuntário, está 
dividido em sistema nervoso simpático e parassimpático, fornece inervação para todos os órgãos do corpo humano, bem como para vísceras, glândulas, músculos lisos e músculo cardíaco. O SNS prepara o organismo para lutar ou para fugir e, quando é estimulado, provoca sinais fisiológicos nas emoções. Nas situações de emergência, o SNS prepara o organismo para a ação por meio da elevação da pressão arterial, frequência cardíaca e respiração ${ }^{14}$.

Todas as funções e influências do SNC no corpo e na emoção tornam-se possíveis graças à presença dos neurotransmissores. A serotonina (5-HT) é um dos neurotransmissores do SNC e os seus níveis cerebrais estão relacionados a alterações de comportamento e humor, ansiedade, agressividade, depressão, sono, fadiga, supressão de apetite etc. Os mecanismos bioquímicos precisos pelos quais os neurônios serotoninérgicos controlam essas funções ainda não estão totalmente esclarecidos ${ }^{15}$.

Em virtude da influência desse neurotransmissor no comportamento, vários estudos têm sido desenvolvidos para verificar a relação de variáveis genotípicas do transportador de serotonina com suicídio, impulsividade/agressividade e os sítios de ligação dos receptores $5-\mathrm{HT}_{2 \mathrm{a}}$ de plaquetas em pacientes deprimidos ${ }^{16-18}$.

É possível também verificar em estudos que emoções como a ansiedade, quando bloqueadas, podem, por meio da influência que exercem no sistema nervoso autônomo, favorecer a crise hipertensiva em determinados pacientes com predisposição genética. $\mathrm{O}$ estresse repetitivo ou uma resposta exacerbada de estresse é um sinal da ativação desse sistema. A atividade simpática na hipertensão está envolvida no índice de morbidade e mortalidade cardiovascular ${ }^{19}$.

Assim, o objetivo do presente estudo é realizar uma revisão bibliográfica de artigos que abordam a relação entre hipertensão e fatores emocionais, levando em consideração a relevância do tema.

\section{MÉTODOS}

Fez-se busca ativa na Biblioteca Virtual em Saúde, na base de dados MedLine (1997-2008), utilizando palavras da língua portuguesa. Com o descritor de assunto "hipertensão", foram disponibilizados 53.389 artigos. Refinando a pesquisa com o descritor de assunto "hostilidade", encontraram-se 28 artigos; com o descritor "raiva", 1 artigo; com o descritor "comportamento impulsivo", não se obteve artigo; com o descritor "personalidade impulsiva", 4 artigos; com o descritor "ansiedade", 142 artigos.

Uma nova pesquisa na base de dados MedLine utilizando o descritor de assunto "doença cardíaca coronária" trouxe 33.608 artigos. Refinando a pesquisa com o descritor de assunto "hostilidade", foram disponibilizados 90 artigos; com o descritor "raiva", não se conseguiram artigos; com o descritor "comportamento impulsivo", não se obteve artigo; com o descritor "personalidade impulsiva", registraram-se sete artigos; com o descritor "ansiedade", foram encontrados 112 artigos.

Entre os estudos pesquisados, selecionaram-se os que relacionavam pressão arterial e doença cardiovascular com os fatores psicológicos. Incluíram-se as doenças cardiovasculares por a hipertensão arterial ser fator de risco para doenças coronarianas e pelos artigos sugerirem raiva, hostilidade e estresse como fatores de risco para hipertensão e outras doenças cardiovasculares. No entanto, não foram selecionados artigos que tratavam exclusivamente de doenças cardiovasculares e fatores psicológicos ou que associavam hipertensão e doenças cardiovasculares com depressão ou doença de Alzheimer.

Pesquisando a bibliografia dos artigos encontrados, foram selecionados estudos, antes de 1997, que traziam conceitos sobre as emoções estudadas, bem como resultados de estudos significativos para a presente revisão.

\section{RESULTADOS E DISCUSSÃO}

Entre os estudos pesquisados, observou-se inconsistência nos achados que relacionam os fatores emocionais com a hipertensão arterial e cardiopatias. Foram encontrados tanto estudos que demonstram relação positiva da raiva, hostilidade, ansiedade e estresse com hipertensão e doenças cardiovasculares quanto estudos que retratam relações negativas. Nos poucos estudos encontrados sobre a impulsividade e as cardiopatias, percebe-se, também, uma dicotomia nos achados. Os dados citados anteriormente estão descritos no Anexo.

Em razão das graves consequências da hipertensão arterial para o organismo humano, pesquisadores têm se dedicado ao estudo de vários agentes de natureza química, física e psíquica capazes de provocar elevação da pressão arterial. Encontram-se na literatura como fatores de risco para a hipertensão arterial: idade, raça, sexo, consumo de sódio, obesidade, fumo, estresse, raiva, hostilidade, impulsividade, ansiedade e depressão. O presente trabalho se propõe a descrever os fatores emocionais e suas associações com a elevação da pressão arterial.

\section{Estresse}

O estresse contribui para grande número de enfermidades, tanto de ordem psíquica como orgânica, e nesta se enquadra a hipertensão arterial.

De maneira genérica, entende-se que o estresse é um conjunto de reações do organismo, caracterizadas pelo desequilíbrio da homeostase, em resposta às ameaças e/ou agressões oriundas de estímulos ambientais, de natureza 
A seguir, citamos algumas pesquisas relevantes que estudaram a influência de fatores psíquicos como hostilidade, estressor e expressão da raiva sobre a pressão arterial sistêmica e doenças cardiovasculares.

O estudo de Yan et al. examinou a relação entre fatores psicossociais (como impaciência, hostilidade, depressão e ansiedade) e o risco de incidência de hipertensão. Os resultados apontam o risco de hipertensão associado com maior tendência à hostilidade. Entretanto, não houve associação de risco em desenvolver hipertensão arterial sistêmica com depressão ou ansiedade. [Yan LL, Liu K, Matthews KA, Daviglus ML, Ferguson TF, Kiefe Cl. Psychosocial factors and risk of hypertension: The Coronary Artery Risk Development in Young Adults (CARDIA) Study. JAMA. 2003;290(16):2138-48.]

O trabalho de Christensen e Smith comparou indivíduos com reduzido nível de ansiedade com aqueles com maior grau de hostilidade. A relação entre hostilidade e pressão arterial foi obtida ao se abordar temas com conteúdo que denotava conflito e hostilidade com outras pessoas, ocasionando maior reatividade cardiovascular. Indivíduos com maior grau de hostilidade exibiram maiores níveis pressóricos relativamente a temas que denotavam conteúdos hostis. Já a utilização de conteúdo neutro nas discussões não resultou em reatividade vascular aumentada. [Christensen AJ, Smith TW. Cynical hostility and cardiovascular reactivity during self-disclosure. Psychosom Med. 1993;55(2):193-202.]

Em 1997, Zhang et al. testaram, em 627 homens de meia-idade, inicialmente não hipertensos, se a supressão da hostilidade prediz incidência de hipertensão. As conclusões relatam que entre os homens de meia-idade ( $\leq 60$ anos) com alta supressão de hostilidade encontrou-se aumento na incidência de hipertensão arterial sistêmica. [Zhang J, Niaura R, Todaro JF, McCaffery JM, Shen BJ, Spiro A 3rd, et al. Suppressed hostility predicted hypertension incidence among middle-aged men: the Normative Aging Study. J Behav Med. 2005;28(5):443-54.]

O estudo de Sheffield et al. não encontrou associação entre reatividade cardiovascular aumentada por estresse, em laboratório, e hipertensão arterial sistêmica. [Sheffield D, Smith GD, Carroll D, Shipley M, Marmot MG. The effects of blood pressure resting level and lability on cardiovascular reactions to laboratory stress. Int J Psychophysiol. 1997;27(2):79-86.]

Lipp, em estudo, testou a hipótese de que o estresse gerado por interações sociais é capaz de aumentar a reatividade cardiovascular e de que a magnitude do aumento dependerá de essa interação envolver conflito ou apoio social. Os resultados mostram que a pressão arterial aumentou durante a entrevista estruturada, sem se registrarem aumentos significativos de frequência cardíaca. [Lipp, MEN. Blood pressure reactivity to social stress in an experimental situation. Rev Cienc Med. 2005;14(4):313-94.]

Sherwood et al., em 1995, avaliaram a base hemodinâmica da hiper-reatividade da pressão arterial influenciada pelo sistema nervoso simpático. Os resultados encontrados mostram que aumentos na pressão arterial foram maiores em 12 homens com hipertensão arterial limítrofe, comparados com 21 homens normotensos de idades comparáveis durante o tempo de reação da ação estressora. [Sherwood A, Hinderliter AL, Light KC. Physiological determinants of hyperreactivity to stress in borderline hypertension. Hypertension. 1995;25(3):384-90.]

Em 2005, Eaker et al. buscaram relacionar tensão e ansiedade à predição de incidentes de doenças cardíacas e fibrilação atrial. Participaram da pesquisa 3.682 pessoas, com idade média de 48,5 anos. Ao final, a tensão caracterizou-se como um fator de risco independente para doenças cardíacas e fibrilação atrial e mortalidade em homens. Sintomas de ansiedade foram associados ao aumento do risco de fibrilação atrial também no sexo masculino. A ansiedade foi considerada um fator de risco para morte em homens e mulheres. [Eaker ED, Sullivan LM, Kelly-Hayes M. Tension and anxiety and the prediction of the 10-year incidence of coronary heart disease, atrial fibrillation, and total mortality: the Framingham Offspring Study. Psychosom Med. 2005;67(5):692-6.]

Outro estudo de Eaker et al., de 2004, relacionou raiva e hostilidade à predição de doenças cardíacas e fibrilação atrial. Participaram da pesquisa 3.873 pessoas, com idade entre 18 e 77 anos. Os resultados apontaram que raiva e hostilidade são significantes preditores de fibrilação atrial em homens, sendo fatores de risco independentes para doença cardiovascular. [Eaker ED, Sullivan LM, Kelly-Hayes M. Anger and hostility predict the development of atrial fibrillation in men in the Framingham Offspring Study. Circulation. 2004;16;109(10):1267-71.]

O estudo de Spicer e Chamberlain, utilizando escalas que aferem expressão da hostilidade e da raiva, concluiu que a pressão arterial em repouso é maior em homens e mulheres que são menos propícios a suprimir a raiva e em mulheres que são mais hostis. [Spicer J, Chamberlain K. Cynical hostility, anger, and resting blood pressure. J Psychosom Res. 1996;40(4):359-68.]

O artigo de Webb e Beckstead relata os resultados de um estudo piloto que testou os efeitos de duas estratégias de intervenção sobre o estresse na pressão sanguínea e estresse - variáveis relacionadas a trabalhadoras americanas negras. Trinta e três mulheres foram divididas em: grupo de meditação cognitiva $(n=10)$, grupo de relaxamento $(n=10)$ ou grupo-controle de tratamento $(n=11)$. Os participantes dos dois grupos de intervenção sobre o estresse participaram do processo por dez semanas; os participantes do grupo-controle receberam monitoramento da pressão arterial durante o período de dez semanas. As mensurações incluíam BP e o Inventário de Expressão de Raiva Traço-Estado (State-Trait Anger Expression Inventory). Os investigadores não encontraram reduções significativas na pressão arterial, raiva ou tensão pessoal nas participantes dos dois grupos de tratamento. [Webb M, Beckstead J, Meininger J. Stress management for African American women with elevated blood pressure: a pilot study. Biol Res Nurs. 2006;7(3):187-96.]

Em pesquisa de 2002, Ohira et al. examinaram a relação da expressão da raiva com a pressão arterial e hipertensão arterial em japoneses. Participaram da pesquisa 4.374 homens e mulheres, com idade entre 30 e 74 anos, de comunidades rurais e urbanas. A escala de expressão da raiva de Spilberger foi utilizada. Nos resultados, observou-se que homens que não expressavam sua raiva provavelmente teriam maior risco de hipertensão arterial, o que não foi observado entre as mulheres. [Ohira T, Iso H, Tanigawa T, Sankai T, Imano H, Kiyama M, et. al. The relation of anger expression with blood pressure levels and hypertension in rural and urban Japanese communities. J Hypertens. 2002;20(1):21-7.]

Contrariando grande parte dos estudos citados anteriormente, a pesquisa de Mann e James não demonstrou associação entre maiores escores de raiva e ansiedade com hipertensão arterial sistêmica. [Mann SJ, James GD. Defensiveness and essential hypertension. J Psychosom Res. 1998;45(2):139-48.]

O artigo de Deter forneceu evidências de maior reatividade cardiovascular induzida por fatores estressores em pacientes com hipertensão arterial essencial, em comparação com pacientes com hipertensão arterial de origem renal e normotensos. [Deter HC, Blecher A, Weber CS. Cardiovascular reactivity of patients with essential and renal hypertension in an emotion-triggering interview. Behav Med. 2007;32(4):117-25.]

Como visto em grande parte dos estudos aqui apresentados, a pressão arterial e a reatividade cardiovascular parecem ser influenciadas por fatores como hostilidade, estressores e raiva, mas mais estudos são necessários para melhor elucidar essas relações. 
psíquica ou física, inusitados ou hostis. Para Selye ${ }^{20}$, estresse designa todos os efeitos inespecíficos de fatores que podem agir sobre o organismo - os agentes estressores capazes de produzir o estresse - que foi caracterizado como uma síndrome de adaptação geral, composta de três fases: reação de alarme; fase de adaptação; fase de exaustão. Para Lazarus e Folkman²1, o estresse psicológico caracteriza-se por uma relação particular entre o indivíduo e o ambiente, que é interpretado pelo indivíduo como um processo de sobrecarga que ultrapassa as suas possibilidades de adaptação e que ameaça o seu bem-estar.

A falta de consenso sobre o conceito de estresse tem causado muitas dificuldades na determinação do verdadeiro papel que ele exerce na epidemiologia das doenças cardiovasculares $^{22}$. No entanto, encontram-se na literatura alguns estudos que demonstram relação válida entre a hipertensão arterial e o estresse mental. Vários estudiosos têm enfatizado a relação entre estresse executivo (laboral) e o aumento do risco de enfermidades cardiovasculares e desenvolvimento progressivo da hipertensão arterial ${ }^{23}$. Em Nova York, estudos de casos e controles foram realizados com 196 empregados vinculados a diferentes tipos de trabalho, constatando-se que 76 sujeitos hipertensos (casos) eram 2,7 vezes mais propensos a ter tensão laboral que os 120 normotensos (controles) ${ }^{24}$. Lindquist et al..$^{25}$ também analisaram a influência, a longo prazo, do estilo de vida, da conduta e do estresse laboral na pressão arterial em homens e mulheres. Todas as conclusões apontam para a necessidade de mudanças nas condutas individuais e de ambiente agradável no local de trabalho, o que pode influenciar beneficamente na variação da pressão arterial.

Pesquisa desenvolvida na Inglaterra com 1.259 homens aponta variações na pressão arterial verificada em hipertensos durante sessões experimentais, em virtude do estresse psicológico ${ }^{26}$. O estudo permitiu concluir que, embora oscilações pressóricas tivessem ocorrido, elas não poderiam justificar a reatividade cardiovascular verificada em inúmeros estudos. O estresse emocional teria sido o principal elemento desencadeador da reatividade observada. Estudos brasileiros conduzidos por Lipp ${ }^{27}$, com paciente hipertensos, e por Lipp et al.28, com adultos, mostram que os hipertensos exibem aumento significativo na pressão arterial quando submetidos a sessões experimentais de estresse emocional. Já no estudo piloto de Webb et al. ${ }^{29}$, com muIheres negras, constatou-se que a eficácia das intervenções para a redução da pressão arterial entre as participantes foi incerta, uma vez que não se obteve redução significativa da pressão arterial delas.

\section{Raiva e hostilidade}

Spielberger et al..$^{30}$ definem raiva como um estado emocional que consiste de sentimentos que variam em intensidade, de leve irritação a fúria. A variação em intensidade desses sentimentos é referida como estado de raiva (state anger), enquanto a frequência com que esses sentimentos são vivenciados é referida como traço de raiva (trait tanger). Quando os sentimentos de raiva são experimentados, eles podem ser reprimidos ou expressados. Para Spielberger et al. ${ }^{30}$, a forma "anger in" refere-se à raiva conscientemente reprimida e "anger out", à raiva expressada. Quanto à hostilidade, sugerem que esta representa um grupo complexo de atitudes negativas que estão relacionadas às emoções tais como cinismo, ressentimento, vingança e alienação.

Estudos têm encontrado relação positiva entre HA e a inibição da expressão de raiva, bem como entre HA e o excesso da expressão de raiva ${ }^{31,32}$. Por sua vez, tem se demonstrado que a hostilidade aumenta o ritmo cardíaco e a pressão arterial ${ }^{33-38}$

Uma pesquisa de metanálise com 45 estudos, publicada em 1996, concluiu que a hostilidade é um fator de risco independente para doença coronariana ${ }^{39}$. Desde então, estudos que investigam a relação entre hostilidade e raiva e doenças cardiovasculares têm demonstrado associações positivas.

Ressalta-se que trabalhos pioneiros desenvolvidos em 1950 e 1960 denominaram o padrão de comportamento tipo A. Nas bases das observações de seus pacientes cardíacos, Friedman e Rosenman ${ }^{40}$ descreveram o indivíduo tipo A como o que é excessivamente inflexível, ambicioso, competitivo, impulsivo e usualmente ansioso.

\section{Ansiedade}

O conceito ansiedade não envolve um critério unitário, principalmente no contexto psicopatológico. A ansiedade pode ser definida como um conjunto de manifestações somáticas - aumento da frequência cardíaca e respiratória, sudorese, tensão muscular, náusea, vazio no estômago, tonteira - e psicológicas - apreensão, alerta, inquietude, hipervigilância, dificuldade de concentração e de conciliação do sono, entre outros ${ }^{41}$. É definida, ainda, como um estado ou sensação de desassossego, agitação, incerteza e temor resultante da previsão de alguma ameaça ou perigo, geralmente de origem intrapsíquica, cuja fonte é desconhecida ou não pode ser determinada ${ }^{42,43}$.

Com base na hipótese de que o estado emocional interfere na variabilidade da pressão arterial, pesquisaram-se os efeitos da felicidade, da raiva e da ansiedade em pacientes com hipertensão arterial borderline e concluiu-se que os três estados emocionais elevam a pressão arterial, encontrando-se forte associação entre a intensidade da ansiedade e a pressão arterial diastólica ${ }^{44}$.

Coryell et al..$^{45}$ expõem que estudos com pacientes com doenças coronarianas e psiquiátricas, bem como amostras baseadas na comunidade, sugerem que transtornos de ansiedade podem estar associados a maior mortalidade, particularmente morte cardíaca repentina, e maior morbidade 
cardiovascular. Evidências anteriores sugerem que pacientes psiquiátricos com transtorno de pânico tinham maior índice de mortalidade. Estudos de Frasure-Smith et al. ${ }^{46}$ associam altos níveis de ansiedade a pior prognóstico e maior recorrência de eventos cardíacos pós-infarto do miocárdio em pacientes coronarianos, embora os achados sejam inconsistentes. Os estudos que examinam a influência da ansiedade no risco de doenças cardiovasculares entre os homens são geralmente positivos, mas a associação entre as mulheres é menor, e algumas evidências clínicas sugerem que a ansiedade pode ser protetora.

No estudo de Muriel et al. ${ }^{10}$, que analisou a relação entre a ansiedade, a personalidade tipo A, descrita como de maior vulnerabilidade ao estresse, e a gênese da hipertensão arterial, não se encontrou associação significativa entre a personalidade tipo A e a hipertensão arterial, mas foi encontrada forte associação entre ansiedade e origem e progressão da hipertensão arterial.

\section{Impulsividade}

Vários são os modelos teóricos que descrevem a impulsividade. Fuentes ${ }^{47}$ agrupa-os em impulsividade como desejo de experimentação, como capacidade reduzida de reflexão e precipitação ao ato e como intolerância à frustração e incapacidade de postergar gratificação.

Segundo Cloninger ${ }^{48}$, a impulsividade está associada a elevada busca por novidades e desprezo por indicadores de perigo ou punição. Já Barratt ${ }^{49}$ destaca a impulsividade por falta de atenção, a impulsividade motora e a impulsividade por não planejamento. Seu modelo teórico relaciona a impulsividade à precipitação ao ato e reduzida capacidade de reflexão. McCrae e Costa ${ }^{50}$ definem a impulsividade como dificuldade de autocontrole, o que incapacita o sujeito a frear seus desejos e adequá-los ao ambiente.

Yan et al..$^{51}$ demonstraram em seu estudo que a impulsividade está relacionada com alto risco cardiovascular e com outros comprometimentos de saúde. No entanto, outros estudos não apresentam resultados tão substanciais.

\section{CONCLUSÃO}

Percebe-se que a associação entre distúrbios emocionais e alterações nas funções viscerais, como a hipertensão arterial, se evidencia quando as estruturas límbicas, responsáveis pelas emoções, são acionadas e produzem respostas cardiovasculares e respiratórias

O que se pode inferir das relações pesquisadas é que o risco de desenvolvimento da hipertensão arterial e a reatividade cardiovascular parecem ser influenciados por fatores emocionais como impulsividade, hostilidade, estressores, ansiedade e raiva. No entanto, mais estudos são necessários para melhor elucidar essas relações.

\section{REFERÊNCIAS}

1. BRASIL. Ministério da Saúde. Secretaria de Vigilância em Saúde. Departamento de Análise de Situação de Saúde. Saúde Brasil 2004 - uma análise da situação de saúde. Brasília: Ministério da Saúde; 2004.

2. Kohlmann Jr O, Guimarães AC, Carvalho MHC, Chaves Jr HC, Machado CA, Praxedes JN, et al. III Consenso Brasileiro de Hipertensão Arterial (CBHA). Arq Bras Endocrinol Metab. 1999;43(4).

3. World Health Organization. Arterial hypertension, Genebra, 1978. (WHO - Technical Report Series, 628).

4. Joint National Committee V - JNC V. The fifth report of the Joint National Committee on detection, evaluation, and treatment of high blood pressure. Arch Intern Med. 1993;153(2):154-83.

5. Dantas J. Patologia cardiovascular relacionada ao trabalho. In: Mendes R. Patologia do trabalho. São Paulo: Atheneu; 2003. p. 1295-328.

6. Diamond EL. The role of anger and hostility in essential hypertension and coronary heart disease. Psychol Bull. 1982;92(2):410-33.

7. Jorgensen RS, Johnson BT, Kolodziej ME, Schreer GE. Elevated blood pressure and personality: A meta-analytic review. Psychol Bull. 1996;120(2):293-320.

8. Krantz DSC. Report of Task Force 1: Psychosocial factors in hypertension. Circulation. 1987;76(Suppl I):184-8.

9. Schneiderman N, Chesney MA, Krantz DS. Biobehavioral aspects of cardiovascular disease: Progress and prospects. Health Psychol. 1989;8(6):649-76.

10. Muriel DMA, Campos JLB, Duque OB, Cuadros VPZ, Echeverri DRP, Ibánez LG, et al. Estudio comparativo del nivel de ansiedad, personalidad tipo A y factores de riesgo asociados a hipertensión arterial en pacientes hipertensos y no hipertensos. Arch Med. 2006;6(13):51-67.

11. Rosenman RH, Friedman M, Straus R, Wurm M, Kositchek R, Hahn W, et al. A predictive study of coronary heart disease. JAMA. 1964;189:15-22.

12. Gallacher JEJ, Sweetnam PM, Yarnell JWG, Elwood PC, Stansfeld SA. Is type A behavior really a trigger for coronary heart disease events? Psychosom Med. 2003;65(3):339-46.

13. Machado ABM. Áreas encefálicas relacionadas com o comportamento emocional. In: Machado ABM. Neuroanatomia funcional. São Paulo: Atheneu; 1985. p. 225-31.

14. Brandão ML. Noções básicas de neuroanatomia. In: Brandão ML. As bases psicofisiológicas do comportamento. São Paulo: Editora Pedagógica e Universitária; 1991. p. 1-11.

15. Bell C, Abrams J, Nutt D. Tryptophan depletion and its implications for psychiatry. Br J PsyC. 2001;178:399-405.

16. Du L, Faludi G, Palkovits M, Bakish D, Hrdina PD. Tryptophan hydroxylase gene 218A/C polymorphism is not associated with depressed suicide. Int J Neuropsychopharmacol. 2000;3(3):215-20.

17. Preuss UW, Soyka M, Bahlmann M, Wenzel K, Behrens S, De Jonge S, et al. Serotonin transporter gene regulatory region polymorphism (5-HTTLPR), [3H]paroxetine binding in healthy control subjects and alcohol-dependent patients and their relationships to impulsivity. Psychiatry Res. 2000;96(1):51-61.

18. Pandey GN, Pandey SC, Janicak PG, Marks RC, Davis JM. Platelet serotonin-2 receptor binding sites in depression and suicide. Biol Psychiatry. 1990;28(3):215-22.

19. Kaplan NM. Systemic hypertension: mechanisms and diagnosis. In: Braunwald E, editor. Heart Disease. A textbook of Cardiovascular Medicine. Philadelphia: WB Saunders; 1997. p. 807-39.

20. Selye H. The stress of life. New York: McGraw-HillBook Company; 1956.

21. Lazarus R, Folkman S. Stress, appraisal and coping. New York: Springer, 1984.

22. Dantas J. Hipertensão arterial e fatores psicossociais no trabalho em uma refinaria de petróleo (dissertação). Universidade Federal de Minas Gerais, Belo Horizonte, 2003.

23. Vrijkotte TGM, Doornen LJP, Geus EJC. Effects on work stress on ambulatory blood pressure, heart rate, and heart rate variability. Hypertension. 2000;35:880-6. 
24. Armario P, Hernández R, Martín-Baranera M. Estrés, enfermedades cardiovasculares e hipertensión arterial. Med Clin (Barc). 2002;119:23-9.

25. Lindquist TL, Berlin LJ, Knuiman MW. Influence of lifestyle, coping and job stress on blood pressure in men and woman. Hypertension. 1997;29:1-7.

26. Sheffield D, Smith GD, Carroll D, Shipley M, Marmot MG. The effects of blood pressure resting level and labiality on cardiovascular reactions to laboratory stress. Intern J Psychophysiol. 1997;27(2):79-86.

27. Lipp MEN. Blood pressure reactivity to social stress in an experimental situation. Rev Cienc Med. 2005;14(4):317-26.

28. Lipp MEN, Pereira MMB, Justo AP, Matos TMG. Cardiovascular reactivity in hypertensives: differential effect of expressing and inhibiting emotions during moments of interpersonal stress. Span J Psychol. 2006;9(2):154-61.

29. Webb M, Beckstead J, Meininger J, Robinson, S. Stress Management for African American Women with Elevated Blood Pressure: A Pilot Study. Biol Res Nurs. 2006;7(3):187-96.

30. Spielberger CD, Johnson EH, Russell SF, Crane RJ, Jacobs GA, Worden TJ. The experience and expression of anger: construction and validation of an anger expression scale. In: Chesney MA, Rosenman RH. Anger and hostility in cardiovascular and behavioral disorders. Washington, DC: Hemisphere; 1985. p. 5-30.

31. Niaura R, Goldstein MG. Psychological factors affecting physical condition. Cardiovascular disease literature review. Part II: Coronary artery disease and sudden death and hypertension. Psychosomatics. 1992;33(2):146-55.

32. Suls J, Wan CK, Costa PT Jr. Relationship of trait anger to resting blood pressure: a metaanalysis. Health Psychol. 1995;14(5):444-56.

33. Lundeberg U, Hedman M, Melin B, Frankenhaeuser M. Type A behavior in healthy males and females as related to physiological reactivity and blood lipids. Psycosom Med. 1989;51(2):113-22.

34. Weidner G, Friend R, Ficarrotto TJ, Mendell NR. Hostility and cardiovascular reactivity to stress in women and men. Psychosom Med. 1989;51(1):36-45.

35. Pope MK, Smith TW. Cortisol excretion in high and low cynically hostile men. Psychosom Med. 1991;53(4):386-92

36. Suarez EC, Harlan E, Peoples MC, Williams RB Jr. Cardiovascular and emotional responses in women: the role of hostility and harassment. Health Psychol. 1992;12(6):459-68.
37. Smith TW. Hostility and health: current status of a psychosomatic hypothesis. Health Psychol. 1992;11(3):139-50.

38. Suls J, Wan CK. The relationship between trait hostility and cardiovascular reactivity: a quantitative review and analysis. Psychophysiology. 1993;30(6):615-26.

39. Miller TQ, Smith TW, Turner CW, Guijarro ML, Hallet AJ. A meta-analytic review of research on hostility and physical health. Psychol Bull. 1996;119(2):322-48.

40. Friedman M, Rosenman RH. Type A behavior pattern: its association with coronary heart disease. Ann Clin Res. 1971;3(6):300-12.

41. Dratcu L, Lader M. Ansiedade: conceito, classificação e biologia. J Bras Psiq. 1993;42(1):19-32.

42. Bleger J. Psicologia da conduta. Porto Alegre: Artes Médicas; 1984.

43. Latorre JM, Beneit PJ. Psicología de la salud. Buenos Aires: Editorial Lumen; 1994.

44. James GD, Yee LS, Harshfield GA, Blank SG, Pickering TG. The influence of happiness, anger, and anxiety on the blood pressure of bordeline hypertensives. Psycosom Med. 1986;48(7):502-8.

45. Coryell W, Noyes R, Clancy J. Excess mortality in panic disorder. A comparison with primary unipolar depression. Arch Gen Psychiatry. 1982;39:701-3.

46. Frasure-Smith $\mathrm{N}$, Lesperance $\mathrm{F}$, Talajic $\mathrm{M}$. The impact of negative emotions on prognosis following myocardial infarction: Is it more than depression? Health Psychol. 1995; 14(5):388-98

47. Fuentes D. Jogo patológico: análise por neuroimagem, neuropsicológica e de personalidade [tese]. São Paulo: Universidade de São Paulo (USP); 2004

48. Cloninger CR. A systematic method for clinical description and classifications of personality variants. Arch Gen Psychiatry. 1987;44:573-88.

49. Barratt ES. Impulsivity: integrating cognitive, behavioral, biological and environmental data. In: McCowan W, Johnson JL, Shure MB. The impulsive client: theory, research and treatment. Washington, DC: American Psychological Association; 1993. p. 39-53.

50. McCrae RR, Costa Jr PT. More reasons to adopt the five-factor model. Am Psychol. 1989;44(2):451-2.

51. Yan LL, Liu K, Matthews KA, Daviglus ML, Ferguson TF, Kiefe CI. Psychosocial Factors and Risk of Hypertension: The Coronary Artery Risk Development in Young Adults (CARDIA) Study. JAMA. 2003;290(16):2138-48. 